\title{
Optical Signature Analysis of Liver Ablation Stages Exploiting Spatio-Spectral Imaging
}

\author{
Mohamed H. Aref ${ }^{1 *}$, Ramy Abdlaty ${ }^{1}$, Mohamed Abbass ${ }^{1}$, Ibrahim H. Aboughaleb ${ }^{2}$, \\ Ayman A. Nassar ${ }^{2}$, and Abou-Bakr M. Youssef ${ }^{3}$ \\ ${ }^{1}$ Biomedical Engineering Department, Military Technical College, Cairo, Egypt \\ ${ }^{2}$ Biomedical Engineering Researcher, Egyptian armed forces, Cairo, Egypt \\ ${ }^{3}$ System \& Biomedical Engineering Department, Cairo University, Giza, Egypt \\ *e-mail: Mh-aref@ieee.org
}

\begin{abstract}
Background and Objective: Thermal ablation modalities such as Radiofrequency ablation (RFA) / Microwave ablation (MWA) are deliberately used for marginally invasive tumor removal by escalating tissue temperature. For precise tumor extinguish, thermal ablation outcomes need routine monitoring for tissue necrosis in a challenging research task. The study aims to exploit hyperspectral imaging (HSI) to evaluate the impact of the liver tissue ablation. Materials and Methods: RFA with temperature range $\left(\geq 80^{\circ} \mathrm{C}\right)$ was accomplished on the ex vivo animal liver and evaluated using a spectral camera $(400 \sim 1000 \mathrm{~nm})$. The spectral signatures were extracted from the HSI data after the following processing steps: capturing three spectral data cubes for each liver sample with total 7-samples (before ablation, after ablation, and after ablation with sample slicing) using an HSI optical configuration. The custom HSI processing comprises "Top-hat and Bottom-hat transform" combined with "watershed transform" image segmentation to increase the intensity for a region of interest (ROI) of the investigated tissue, linking spectral and spatial data. Additionally, statistical analysis for HSI data was performed to exclusively select the best spectral band that discriminates between the normal, thermally-damaged, and ablated liver regions. Results: The variation of the optical parameters for the investigated liver samples provides variable interaction with the light diffuse reflection $\left(R_{d}\right)$ over the spectrum range $(400 \sim 1000 \mathrm{~nm})$. Where, the extracting spectral information of the various tissue zones from the induced RFA linked to the hemoglobin, methemoglobin, and water permits variations. The generated spectral image after image enhancement utilizing "Top-hat and Bottom-hat transform" followed by "watershed segmentation", showed high contrast between normal and thermal regions at a wavelength $(600 \mathrm{~nm})$. However, the wavelength $(900 \mathrm{~nm})$ shows a high variance between the normal and ablated regions. Finally, delineation of the thermal and ablated regions on the complemented enhanced image. Conclusion: HSI is considered a promising optical noninvasive technique for monitoring the RFA toward enhancing the ablation-based treatment for liver tumor outcomes. (C) 2021 Journal of Biomedical Photonics \& Engineering.
\end{abstract}

Keywords: liver cancer; hyperspectral imaging; radiofrequency ablation; thermal damage; top-hat and bottom-hat transform; watershed segmentation.

Paper \#3412 received 29 Mar 2021; revised manuscript received 19 Jun 2021; accepted for publication 21 Jun 2021; published online 29 Jun 2021. doi: 10.18287/JBPE21.07.020306. 


\section{Introduction}

The liver is the biggest organ regarding the human organs and exemplifies about $3 \sim 5 \%$ of its weight in grown-ups. The growing knowledge of the liver anatomy assists in developing novel surgical techniques that are mainly used in treating both primary and secondary liver lesions [1]. There are several causes of liver inflammation, such as (drug- venomousness, hepatitis A, hepatitis B, liver alcoholic disease, and nonalcoholic steatohepatitis) [2].

Hepatocellular carcinoma (HCC) is the significant recurrent crucial tumor of the liver [3]. HCC is ranked the $4^{\text {th }}$ in tumor repetition frequency and the $3^{\text {rd }}$ in death by malignancy worldwide [4]. HCC is the most widely recognized liver cancer with more or less three-quarters of the diagnosed cases [5]. Over 600,000 liver patients pass away by HCC annually. Seeking the causes for the liver malignancy is a challenging task for both clinical and pharmaceutical research teams [6]. Egypt witnessed a noticeable augmentation of HCC patients who were earlier diagnosed with other liver diseases regarding the other countries in the same region [7].

The early diagnosis of HCC enables the request of primary stage therapies that considerably increases the potential for patients survival [8]. Liver transplantation, if available, is the highest survival option for HCC patients treatment [9-11]. The availability of liver transplantation is restrained by the availability of donors and the financial abundance beside complying with the Milan criteria [12].

The fusion of the significance of minimum invasive diagnosis and therapeutic methods is an approach for elevating the proficiency of the modern surgical techniques [13]. Although, the resection of cancers in neurosurgery with regular restorative strategies are generally depends on the experience of neurosurgeons during the open operation [14]. However, for liver-tumor therapy, careful resection is a very common technique [15]. The third therapeutic option is suitable for primary stage HCC patients with the non-cirrhotic liver is hepatic resection [16]. Minimal invasive thermal ablation modality has proved to be a vital treatment choice when achievable [17].

The minimal invasive thermal ablation techniques such as: radio-frequency ablation (RFA) [18], laser thermal ablation (LTA) [19,20], microwave ablation (MWA) [21,22], high-intensity focused ultrasound (HIFU) [23], and bulk thermal ablation (BTA) [24, 25]. All the aforementioned techniques represent significant result therapy for unresectable essential and secondary liver tumors $[18,26,27]$. The main role of all the thermal ablation techniques is to gradually increase the temperature in the investigated sample $\left(\geq 60^{\circ} \mathrm{C}\right)$ to deliver a necrotic condition for tumor cells.

Recently, various studies informed the incorporation of few imaging techniques for the purpose of monitoring the ablation of the liver's tumors. Where, the diffuse reflectance spectroscopy (DRS) is utilized to assess RFA of 8 investigated samples of liver tumor with high accuracy (97 99\%) to discriminate between the predicted thermal damage and the histology report [17]. Moreover, another study exploiting the computed tomography (CT) with MWA to investigate three ex-vivo porcine samples with a thermal procedure $(100 \mathrm{~W}$, $4.5 \mathrm{~min}$ ), the results demonstrate ablation region $(4.1 \pm 0.2 \times 5.6 \pm 0.2) \quad$ [28]. Additionally, Magnetic Resonance Imaging for Thermometry (MRIT) is recently counted as the gold-standard in measuring the tumor necrosis by thermal ablation [29].

HSI is else called imaging spectroscopy, which declares the innovation of integration typical imaging and spectroscopy modalities to secure both spatial and spectral data of an item [30-32]. Although imaging spectroscopy has been accessible as a far off detecting innovation since the 1980s [33,34]. Up to this point, spectral imaging has regularly just been accessible to a constraint number of researchers and experts because of the significant expenses of building spectral cameras and the complexity of processing spectral extracted data related to multiple bands [35-39].

HSI is an expanding imaging technique to divergent fields of science including the medical field such as monitoring of thermal ablation.-M. Landro et. al. present a study in assessing the laser ablation in the porcine in vivo liver [40] and monitoring laser-induced thermal damage in gastric mucosa [41]. There are also Skin Erythema and Pigmentation [42], Breast cancer identification from the pathological tissues [43-46]. Furthermore, a research on brain tissue was capable to identify glioblastoma (GB) with sensitivity $88 \%$ and specificity $77 \% \quad[47,48]$. Moreover, the diffuse reflectance measurements to indicate the hemoglobin oxygenated and deoxygenated condition [49-51].

The incident light interrelates with the liver tissue regarding its optical properties (type/size/density/color) to provide several optical properties as example (Transmission, Absorption, and Diffuse Reflectance) $[52,53]$. As a result of these properties, it can be recognizing and characterized the investigated samples in a certain band by their spectral signature, as more clarified in Fig. A1 in the appendix.

This study was performed in the form of successive series of investigation trials to highlight HSI capability to monitor RFA [54-56]. M. H. Aref et al. have showed in previous study that the HSI could be discriminate the surface thermal removals upon on the investigated 10 samples of the ex vivo bovine liver at ideal spectral picture $(720 \pm 18.92 \mathrm{~nm})$ [55]. Additionally, investigate both the side penetration and surface of RFA [54]. Moreover, successfully distinguish and delineate with cross-correlation algorithm the thermal damage in ex vivo liver samples [56]. However, the HSI strategy offers a particular spectral picture over the spectrum range as it gathers latitudinal and spectral data for under investigation samples [8, 57-58].

In the presented study, we aimed to build up an optical imaging system to measure the diffuse reflectance $(R, d)$ exploiting the hyperspectral (HS) camera to identify and characterize the spectral signature of the thermal and 
ablated regions in ex vivo liver samples. Applying image processing incorporates contrast enhancement to the raw data uitilizing "Top-hat and Bottom-hat transform" combined with "watershed transform" image segmentation to increase the intensity of the region of interest (ROI) for the investigated tissue. Moreover, delineation of the thermal and ablated regions on the complemented enhanced image to provide a vital information for assisting the surgeon in monitoring the thermal ablation outcomes.

\section{Materials and Methods}

The captured image data with the HSI system, image progression in advance, and subsequently thermal ablation, contrast enhancement to the original image using "Top-hat and Bottom-hat transform" combined with "watershed transform" image segmentation algorithm as channels to highlight the thermal and ablated regions in the scan image cubes by the HS camera. The capturing time for each image is about $6 \sim 10 \mathrm{sec}$ and calculation time is $<15 \mathrm{sec}$ using MATLAB software (The MathWorks, Natick, MA, USA, 2019) on a computer with processor Intel Core i7@1.8 GHz with a 16 GB RAM.

\subsection{The prospective approach interconnections protocol and procedures}

- Sample preparation \& Tissue characterization.

- Hyperspectral (HS) scan Images for the ex vivo liver samples.
- The measurements for sample diffuse reflection $\left(R_{d}\right)$.

- The statistical Calculation of the optical properties for validation.

- Selecting the optimum spectral image with high contrast between normal, thermal, and ablation.

- Applying the Custom clustering algorithms.

- Delineation of the ablation and thermal effect regions of the enhancement image.

\subsection{Investigated sample slicing and preparation}

Before proceeding to the experimental investigation, the sample collection procedure was approved by Ain Shams university - faculty of medicine - ethics committee. A total of seven different samples of bovine liver tissue achieved from a fresh slaughtered cow which was attained from a local abattoir in Egypt, then transported in an icebox to the laboratory. The investigated ex vivo liver samples were crudely cut into slices with approximate sizes $(\sim 10 \times 8 \mathrm{~cm})$, Sample thickness $23 \sim 36 \mathrm{~mm}$. The investigated samples were preserved in an evacuated bags in the freezer at temperature $\leq-65^{\circ} \mathrm{C}$, and picked out and added in a neutral buffered saline before the RFA experiments with enough time [55]. The inspected lap trails at temperature $23 \sim 24^{\circ} \mathrm{C}$, and the explored sample temperature $25 \sim 28{ }^{\circ} \mathrm{C}$, observed with a multimeter (Fluke, 289, USA), as shown in Fig. 1 and the additional investigated samples had been displayed in Fig. A2 in the Appendix.
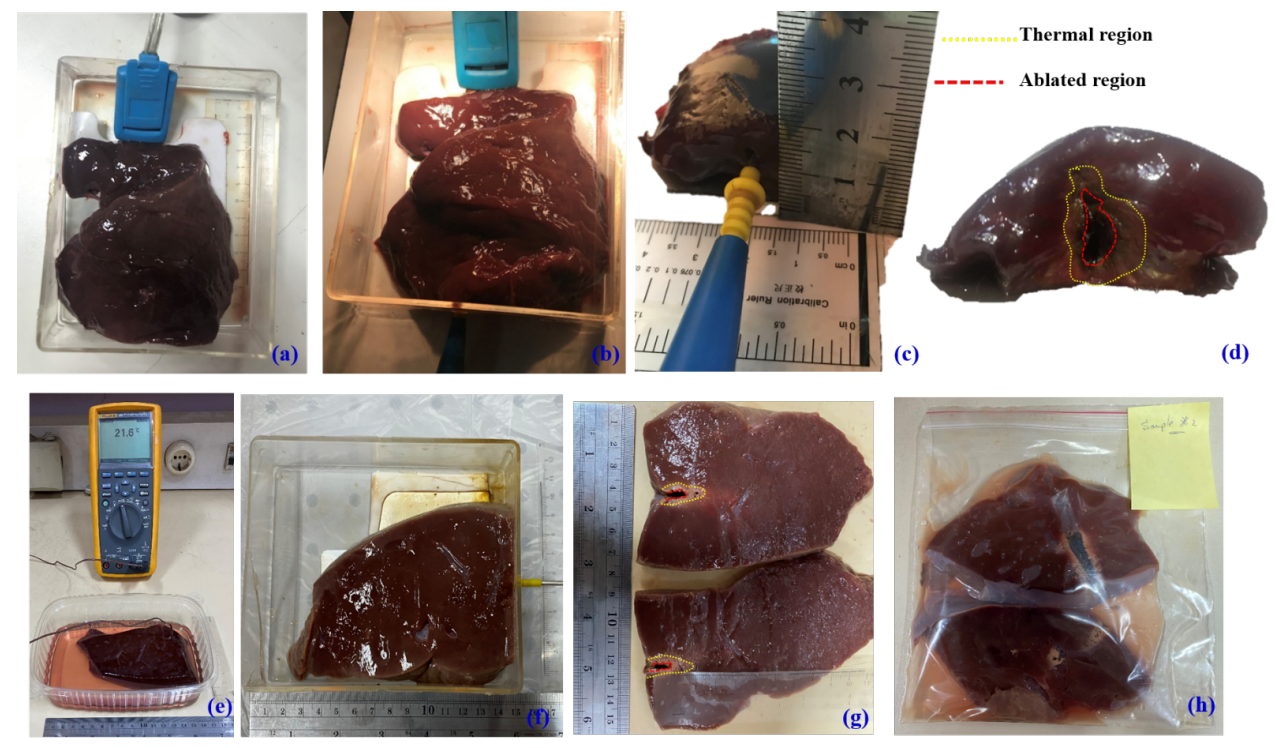

Fig. 1 The investigated ex vivo liver samples; (a) the liver Sample \#1 before ablation, (b) the liver Sample \#1 after ablation in the plastic tray of the experiment and with the RF probe, (c) the illustration for the active blade of the RF tip inserted in the investigated liver Sample \#1; (c) the liver Sample \#1 after the ablation trail highlighting the affected ablation region in Red dotted lines and thermal regions in yellow dotted lines, (e) the investigated liver Sample \#4 before ablation inserted in neutral buffered saline and observed with a multimeter (Fluke, 289, USA), (f) the investigated liver Sample \#4 after ablation in the experimental plastic tray and with the RF tip inserted, (g) the investigated liver Sample \#4 after ablation and slicing to compare the actual ablated region with scientific expert, (h) the sliced liver Sample \#4 after ablation and wrapped in the vacuumed bags and tissue storage solution prepared to be sent to the pathological lap. 


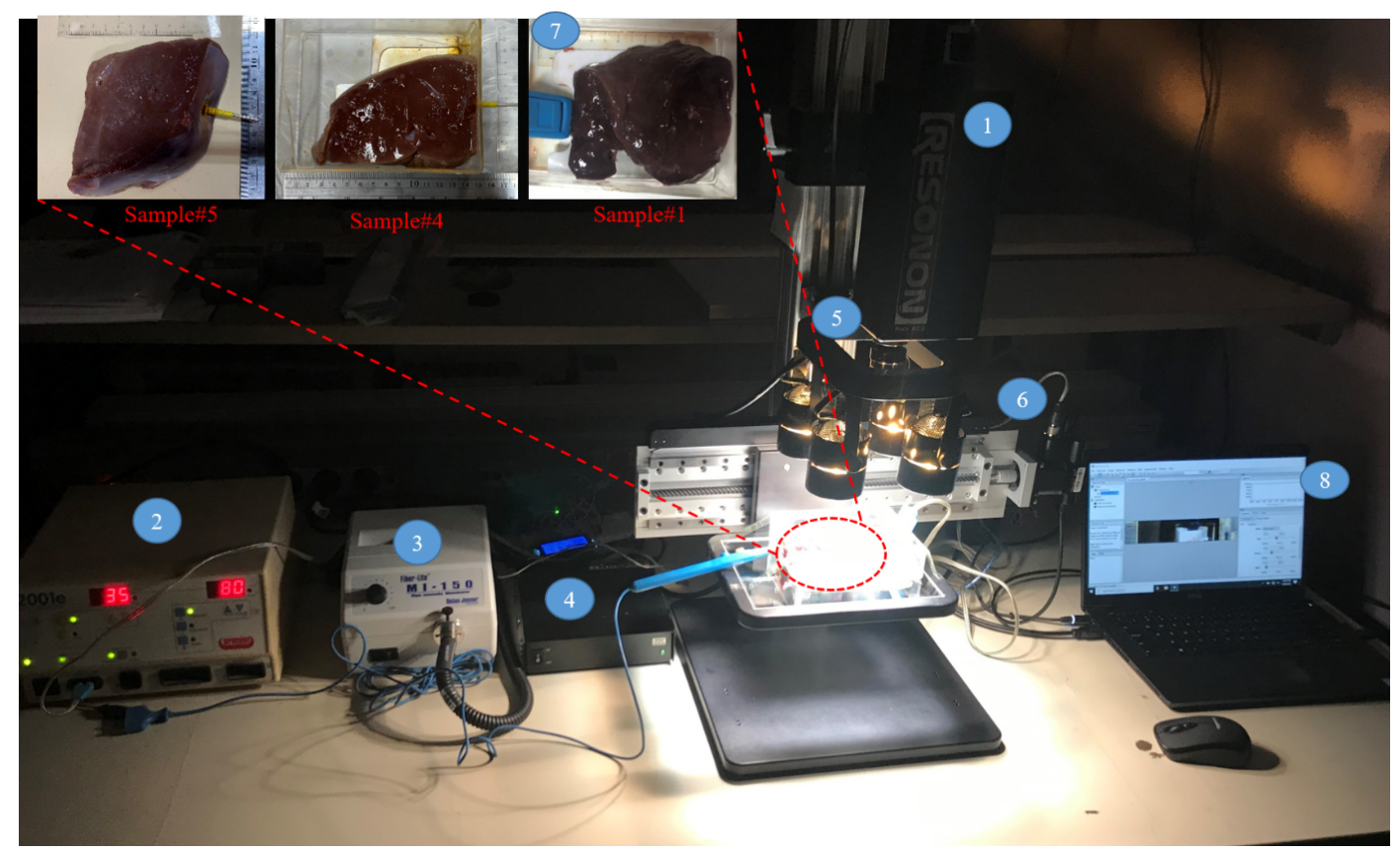

Fig. 2 Hyperspectral optical imaging system for measuring the light diffuse reflectance $\left(R_{d}\right)$ of the investigated ex vivo tissue samples; (1) hyperspectral camera (Resonon, Pika XC2, USA); (2) electrosurgical generator (Premier; 2001e, France); (3) the source light for the transmission measurement trails (Fiber-lite, MI-150, USA); (4) the power supply controller for the light source (Mean Well, ENP360-12, Taiwan) of diffuse reflection $\left(R_{d}\right)$ measurement trails; (5) the 4-halogen lamps $(4 \times 35 \mathrm{~W}$ halogen lamps) at wavelength range $(380 \sim 1050 \mathrm{~nm})$; (6) the linear translation stage; (7) the investigated ex vivo liver samples; (8) the computer and image software processing and analysis.

The experiment was performed with Radiofrequency (RF) Generator (Premier; 2001e, France) to generate the necessary thermal ablation in the liver tissue. The power protocol was as following $(25 \sim 50 \mathrm{~W}, 1 \sim 5 \mathrm{~min}$, Continues). The affected ablation region in the sample was around $\sim 10 \times \sim 8 \mathrm{~mm}$. Furthermore, all the image processing of the captured HS cube was less than 5 min during the thermal elaboration. The complete data of the investigated tissue with the power protocol for each sample experiment, had been illustrated in Table A1 in the Appendix.

\subsection{Optical imaging system and experimental trail}

The active blade of the RF generator was positioned in the center of the investigated sample. Where terminal was embedded around $1.5 \sim 2 \mathrm{~cm}$ into the liver sample, and RF power was applied for $2 \sim 5 \mathrm{~min}$. Following data procurement, markers were embedded into the imaging plane on the two sides of the electrode, and the ablation was cut through this plane and captured with the commercial CCD camera for data recording. After the RFA the samples is sliced in the coronal plane from the RF tip insertion and had been manually measured and prepared to be sent to the pathology evaluation (pathology is the ground truth to validate the tissue necrosis and thermal effect).

To capture the necessary HSI data, we exploited hyperspectral camera (Resonon, Pika XC2, USA) with spectral resolution equal to $1.3 \mathrm{~nm}$, bit depth of 12 bits, maximum frame rate equal to $165 \mathrm{fps}$, spatial channels $=1600$, and 462 spectral channels. The camera is incorporated with a lens (Schneider, $6 \mathrm{~mm}, \mathrm{CNG}$ 2.1/6- 0901, Range $400: 1000 \mathrm{~nm}$, Germany). The utilized light assembly for the HSI data capturing is a configuration of a polychromatic source light controller (Mean Well, ENP360-12, Taiwan) with a light source $(4 \times 35 \mathrm{~W}$ tungsten halogen lamps) at wavelength range $(380 \sim 1050 \mathrm{~nm})$. The prospective approach and its experimental setups aimed to measure the light diffuse reflectance $(R, d)$ for an ex vivo tissue sample, by exploring the optical properties spectroscopy in the near-infrared and visible (NIR-VIS) spectrum, as shown in Fig. 2.

\subsection{Hyperspectral Image Conquering}

HSI sensors produce a three-dimensional (3D) information structure, called HS cube, where the spatial data is contained in the initial two measurements, while the third measurement incorporates the spectral data $[59,60]$. In a hyperspectral picture, every pixel has an arrangement of reflectance in various spectral frequencies that can show the spectral outline of that pixel [48].

Three individual arrangements of hyperspectral pictures were gotten for each explored liver sample. Line-scan images were captured for exposure times of $6 \mathrm{sec}$ at $1.3 \mathrm{~nm}$ intervals, and corresponding to $1600 \times 600$ pixels per spectral band $(3.6 \mathrm{sec}$ for each cube image). The hyperspectral images were composed of a total of 447 spectral bands in the range from 
approximately 379 to $1050 \mathrm{~nm}$, incorporated with a lens with Visible and Near-infrared range (VNIR) range (400 to $1000 \mathrm{~nm})$.

\subsection{Basic Concepts and Fundamentals of Clustering Algorithms}

The cluster is commonly handled as a gathering of an item's which were "identical" between them just as "divergent" to an item's having a place with the further clusters [61]. Clustering algorithms are as follows:

1. Exclusive-clustering.

2. Overlapping-clustering.

3. Hierarchical-clustering.

4. Probabilistic-clustering.

In this study, we used one of the capturing is frequently applied clustering and segmentation algorithms:

- K-mean clustering.

- Watershed transform / Segmentation.

- $\quad$ Fuzzy C-means clustering.

\subsubsection{K-means Clustering}

The K-means clustering is the simplest unsupervised learning algorithm that is capable to solve the distinguished clustering issues. K-means algorithm is prevalent in rapid decision making approach for its simplicity, adequacy as well as being moderate although it had a steady presentation across various issues $[54,62,63]$ Despite the way that the time inconvenience was direct to the information size, customary k-implies is so far not admirably compelling to manage a web-scale data [61]. The strategy follows a basic as well as a simple approach to characterize a given data set via a specific number of clusters settled an earlier [64].

\subsubsection{Watershed transform / Segmentation}

The watershed transform is a structural essential tool for image segmentation [65]. It is established on a mathematical structure for segmenting the data of interest. It was initially represented by L. Vincent and Soille in the image segmentation field then extended rapidly in recent years $[66,67]$. It is touchy to weak edges and is appropriate for getting one-pixel associated and closed contours with accurate area [68, 69]. Conventional watershed segmentation is sensitive to noise and can prompt critical over-segmentation. Along these lines, numerous analysts have proposed different strategies ceaselessly for improving the technique including developing a preprocessing step for calculating the distance transform for a binary picture before watershed transformation $[67,70]$.

\subsubsection{Fuzzy C-means clustering}

Fuzzy C-means (FCM) strategy is considered as a significant high beneficial because it isolates a lot of information from one single picture unlike to other hardsegmentation methods [71]. In FCM clustering technique, the image pixel was assigned to the fuzzy clusters instead of a label [72]. FCM empowers pixel to have a spot with different clusters through changing degrees of part transport function, dissimilar to a hardclustering system that powerfully designates the pixel to just a single class. The FCM Algorithm is the technique for clustering at which it concurs a solitary purpose of a content having a place with two or more clusters [73].

Maximize Contrast

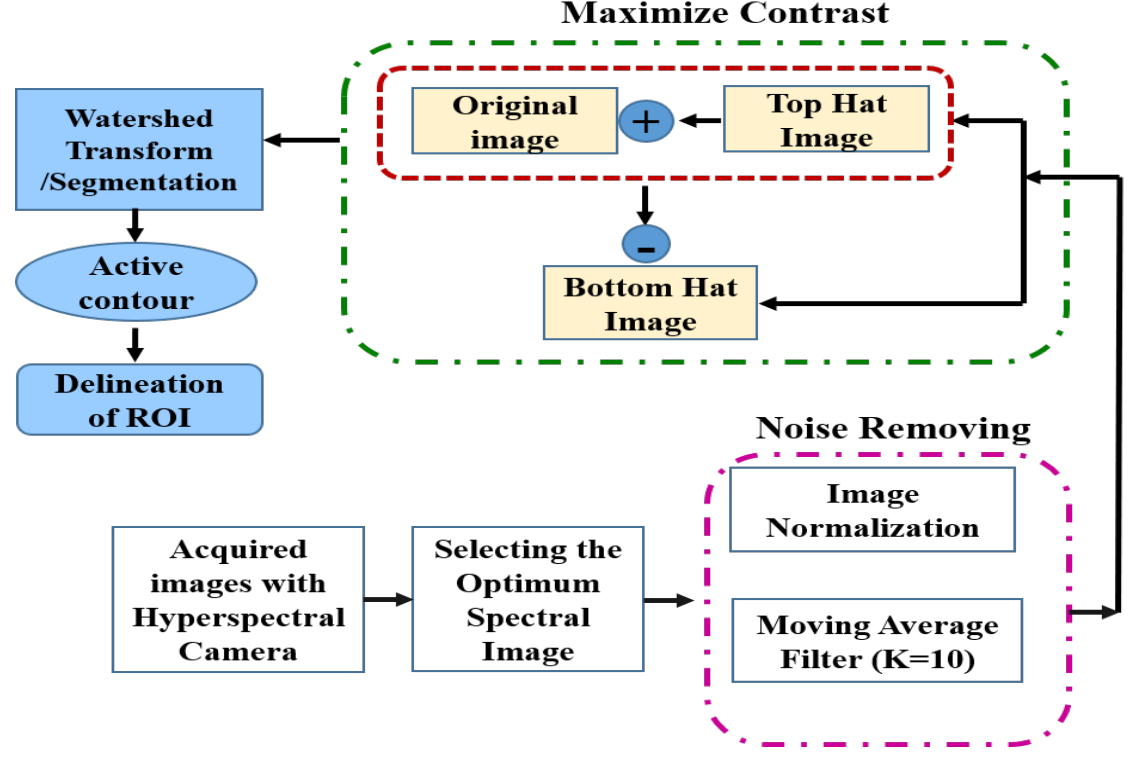

Fig. 3 The basic block diagram of the custom algorithm for image analysis and preprocessing after selecting the optimum spectral image of the investigated liver sample tissue. 


\subsection{The Image Analysis and Preprocessing}

The image analysis and preprocessing step mainly incorporates the Moving average filter and normalization for the acquired HSI to remove the background noise. Moving average filter $(\mathrm{K}=10)$ is exploited to banish the noise influences. For image enhancement, we exploit a common methodology using "Top-hat and Bottom-hat transform". Next, utilizing the "watershed transform" for image segmentation to increase the ROI of the investigated liver tissue samples. Moreover, delineation of the thermal and ablated regions on the complemented enhanced image to discriminate the distinct regions, as explained in the block diagram in Fig. 3.

A basic advance in HSI imaging, before image procurement, is a level field correction for data standardization. A white equalization and dim current measurements were utilized to gain relative reflectance from the sample [60]. The dark cube was captured by closing the HS camera lens with its cap to avoid any incidence light to the sensor. Information from a dark image and white balance estimations were utilized to correct the deliberate material image. The fundamental reason for this amendment is to wipe out artifacts and noise impacts on the sample tissue, as more clarified in Eq. (1):

$$
R F(\theta)=\frac{\operatorname{Im}(\theta)-\operatorname{Id}(\theta)}{\operatorname{Iw}(\theta)-\operatorname{Id}(\theta)} \times 100 \%
$$

where $R F(\theta)$ is the qualified reflectance of the sample image, $\operatorname{Im}(\theta)$ is the caught image, $\operatorname{Id}(\theta)$ is the dark scanned image with a closing lens, and $\operatorname{Iw}(\theta)$ is the acquired whiteboard image.

Employing the normalization on the acquired image to remove the polychromatic light noise. The originally captured images numerous due to the incident light, the asymmetrical shape of the investigated sample, and temperature deviations, spectral images have to be normalized comprising pixel normalization, as demonstrated in Eq. (2).

$$
\begin{aligned}
& I_{\text {new }}=\left(I_{\text {previous }}-\min _{\text {previous }}\right) \times \\
& \times \frac{\max _{\text {new }}-\min _{\text {new }}}{\max _{\text {previous }}-\min _{\text {previous }}}+\min _{\text {new }} .
\end{aligned}
$$

Even though, Normalization adjusts an m-dimensional grayscale subsequent image $I_{\text {previous }}:\{A\}$ with intensity evaluations in the range $\left(\right.$ min $\left._{\text {previous }}\right)$ to the extreme ( $\left.\max _{\text {previous }}\right)$ into a new image $I_{\text {new }}:\{A\}$ with intensity rates in the least range $\left(\min _{n e w}\right)$ to the extreme $\left(\max _{n e w}\right)$.Then, utilizing the Moving average filter, the filter at kernel value $=10$ for noise elimination and image improvement [74], as clarified in Eq. (3).

$$
f(x \times y)=\frac{1}{q t}+\sum_{(r \times c) \in W}^{\infty} S(r \times c),
$$

where $S$ is the noisy picture, $f(x \times y)$ is the restored picture, and $r$ and $c$ for the row and column coordinates correspondingly, within a window $W$ of size $q t$ where the procedure takes place.

To maximize the image contrast, we utilized Top-hat and Bottom-hat Transform for image contrast enhancement.

- The top-hat transform could be illustrated as the difference between the original image and the opening image. Where the opening image is a collection of spotlight parts that fit a precise structuring element.

- The bottom-hat transform could be illustrated as the difference between the closing of the original image and the original image. The closing of an image is the collection of background parts of an image that fit a precise structuring element.

Although in image contrast enhancement, the bottomhat image represents the gaps between the targets of interest. To maximize the contrast between the targets and the gaps that separate them from each other, the "bottom-hat" image is subtracted from the "original + top-hat" image.

Since "watershed transform" distinguishes intensity "valleys" of the acquired image. We utilized the (imcomplement) function on the contrast-enhanced image to convert our targets of interest to intensity valleys. We discriminate all the intensity valleys under a certain threshold with the (imextendedmin) function. The outcome of this function is a binary image. Where the location is more important than the region size of the image. To confine the selected valleys extracted by the function (imextendedmin) we utilized another function called (imimposemin) changing the valley`s pixel value to " 0 ". However, all the discrete regions contain the minimum values will be detected by the "watershed transform".

Finally, the reflectance spectra of the pixels made out of thermal ablated tissue surface were isolated and used to figure a typical reflectance extend to delineate the thermal levels in the investigated liver sample. The delineation contours were finally displayed on the complemented enhanced image in red and blue for ablation and thermally affected areas, respectively.

\section{Results}

Regarding the system interconnections protocol and process, beginning from hyperspectral image scanning for the investigated liver sample and image enhancement to measure the $\left(R_{d}\right)$ of the various regions of the investigated sample, we could differentiate between these regions regarding the wavelength variations. each pixel in the investigated liver tissue has a relative reflectance for the wavelength variations. The experimental setup was evaluated and verified with respect to the pathological report. 


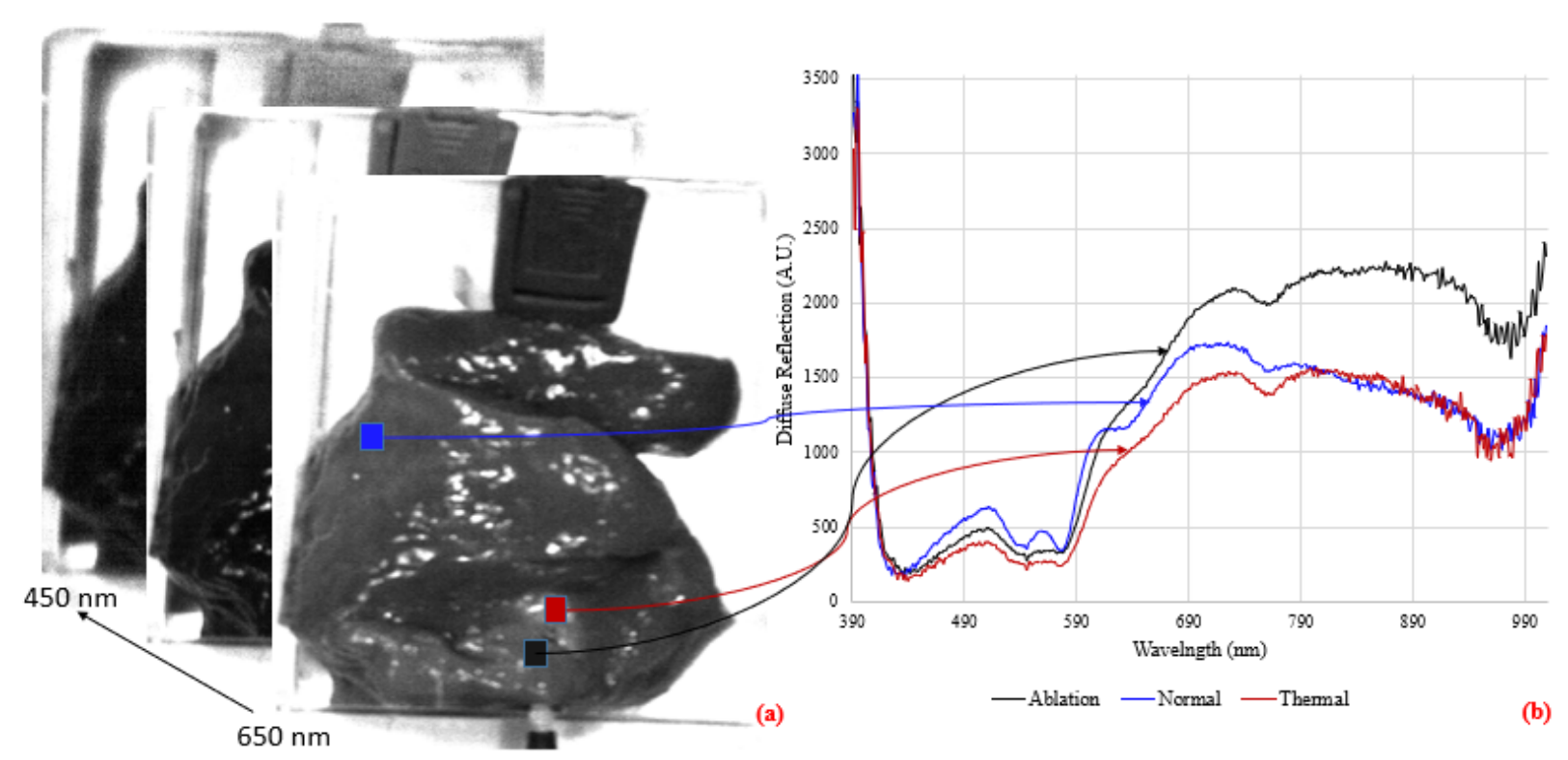

Fig. 4 (a) The scanned Hyperspectral (HS) image of the investigated Liver Tissue Sample\#1 highlighting the measured diffuse reflection $\left(R_{d}\right)$ regions over the spectral range $(400 \sim 1000 \mathrm{~nm})$; (b) the blue pixel demonstrate the $\left(R_{d}\right)$ of the normal tissue not affected with the thermal region where it's presented in the graph by the blue solid line; the red pixel representing the thermal regions and plotted in the graph with the red solid line, additionally the black pixel represent the ablated region with a black solid line in the graph.
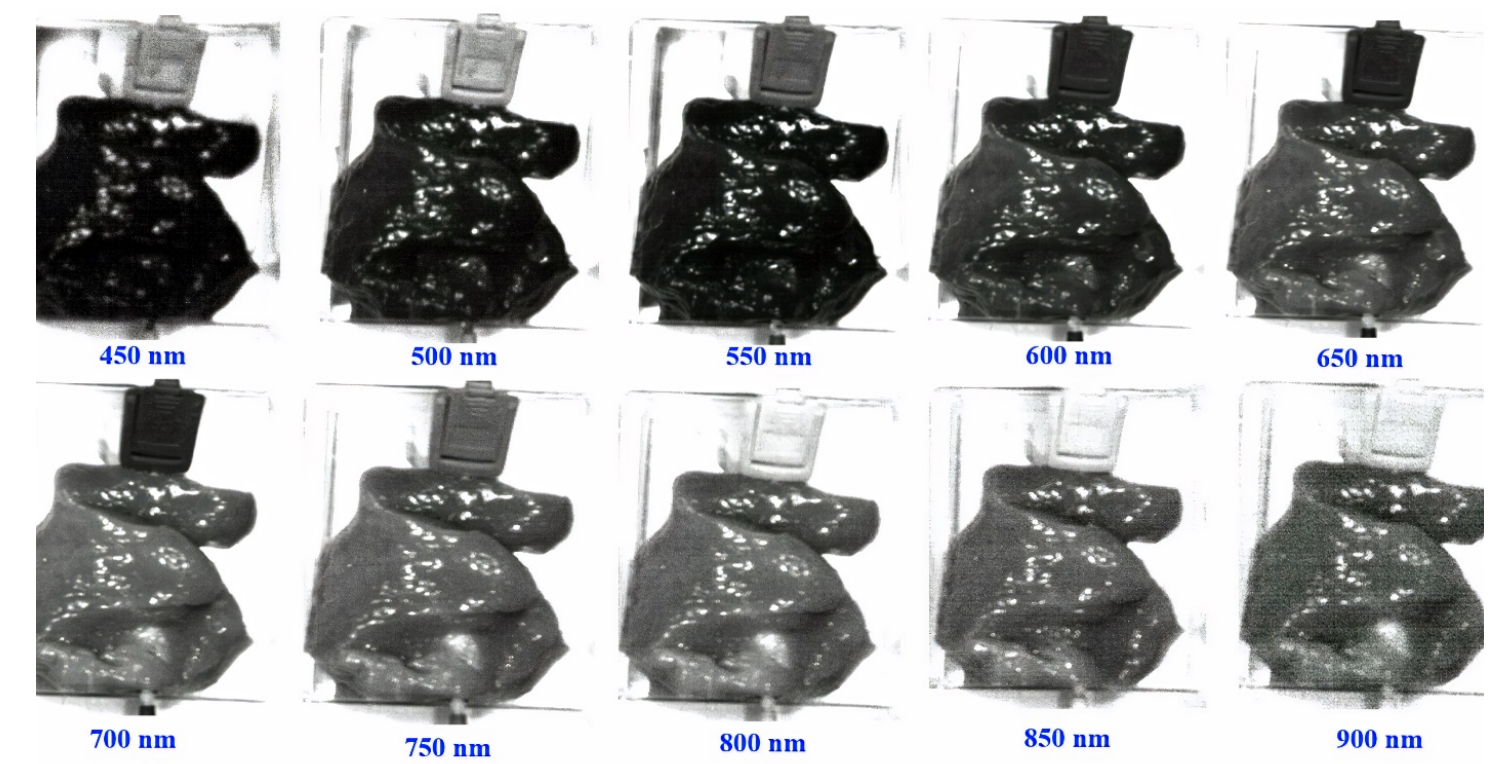

Fig. 5 The scanned HS images of the investigated ex vivo liver Sample \#1 after ablation from $450 \mathrm{~nm}$ till $900 \mathrm{~nm}$ with $50 \mathrm{~nm}$ resolution, where we could visually see the differentiation of the Ablation and thermal effect regions on the various HS images in the spectral range.

The reflectance spectrum signature of each pixel generated from the thermal and ablated regions of a liver tissue surface was separated and used to compute a normal reflectance range. The degree and level of thermal effect to the tissue could be highlighted according to the spectrum wavelength to distinguish the selective regions (ablated, thermal manipulated, and normal tissues). The spectral reflectance signatures were measured and evaluated from the mean of the three different regions on the investigated liver tissue sample, as illustrated in Fig. 4(a).
The deliberate optical spectrum of the investigated liver sample over the wavelength spectrum $400 \sim 1000 \mathrm{~nm}$. The experiment was exploited utilizing ex vivo bovine liver tissue, where captured HSI is scanned and segmented, selecting specific regions to measure the optical properties of the ablation region and another region around it for measuring the thermal consequence to the normal tissue, as shown in the pixel identification in Fig. 4(a). 


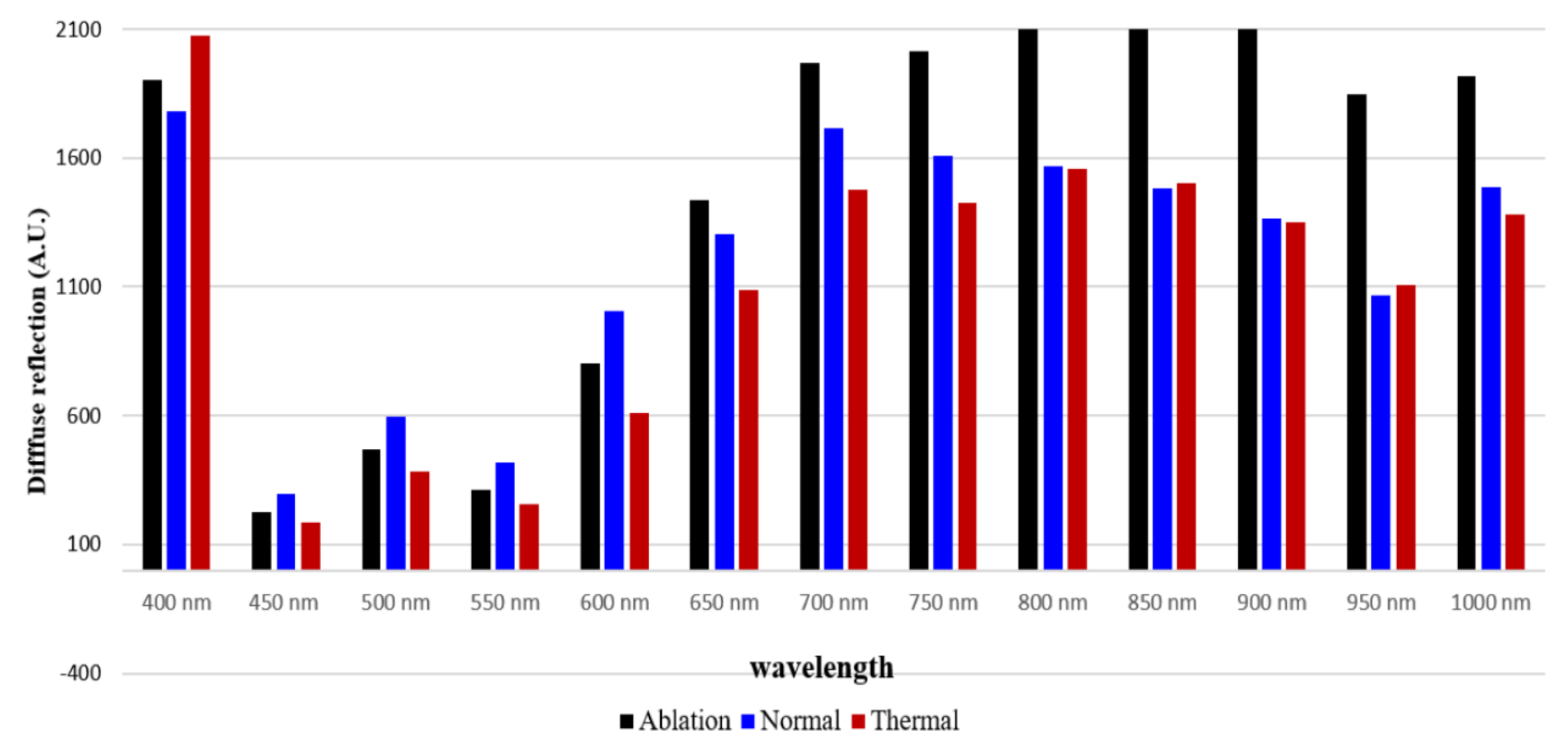

Fig. 6 The bar plot which highlighted the contrast between each region (normal, thermal, and ablated) districts in the investigated ex vivo Liver Sample \#1.

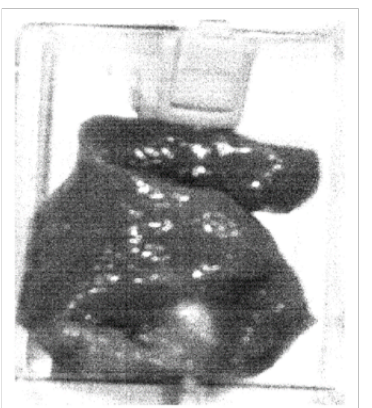

(a) Original Image

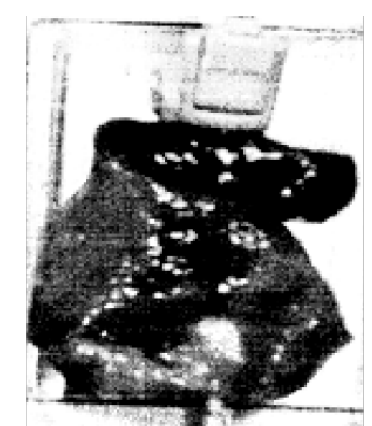

(b) Contrast Enhanced Image

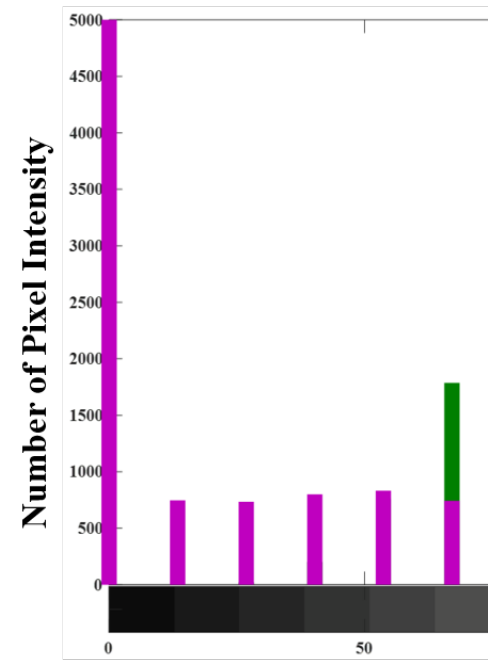

(c)

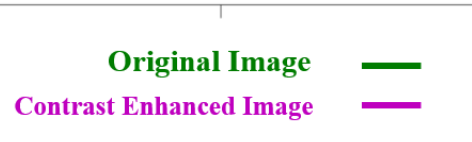

Fig. 7 (a) The original image at wavelength $900 \mathrm{~nm}$ of the investigated liver tissue Sample \#1 before image contrast enhancement; (b) the enhanced image contrast utilizing the Top-hat and Bottom-hat transforms; (c) the histogram which reveals the contrast of the original image with the green bars and the enhanced image contrast with the purple bars.

The measured diffuse reflectance $\left(R_{d}\right)$ of the selected regions (normal, thermal, and ablated) were plotted in the graph illustrating the distinction shift of the wavelength between the normal tissue and ablated signature, as demonstrated in Fig. 4(b). The blue pixel demonstrates the diffuse reflection $(R, d)$ of the normal tissue which is 
not affected with the thermal region where it is presented in the graph by the blue solid line; the red pixel representing the thermal regions and plotted in the graph with the red solid line, additionally the black pixel represent the ablated region with a black solid line in the graph.

To avoid the time-consuming of the 447 spectral images of the investigated sample, we select the Ten images of the investigated sample after ablation over the spectrum $(400 \sim 1000 \mathrm{~nm})$ with $50 \mathrm{~nm}$ resolution. We noticed visually that to differentiate between the normal and the thermal region was more identified in the spectrum $(550 \sim 650 \mathrm{~nm})$. However, to segregate between the normal and the ablated regions was highlighted in the wavelength $(850 \sim 950 \mathrm{~nm})$, as liver Sample \#1 illustrated in Fig. 5, liver Sample \#4 in Fig. A3, and liver Sample \#5 in Fig. A4 at the Appendix.

The bar chart highlighted the contrast between the investigated ex vivo liver sample regions (normal, thermal, and ablated). The wavelength $(600 \mathrm{~nm})$ shows high contrast between the normal and the thermal regions. On the other hand, at wavelength $(900 \mathrm{~nm})$ represents high contrast between the normal and the ablated regions, although discriminate between the thermal and ablated regions, as more clarified in Fig. 6.

Selecting the optimum spectral image $(900 \mathrm{~nm})$ from the bar plot of Fig. 6, which could discriminate between (normal / ablated) regions, additionally between (thermal / ablated) regions to the original image utilized with the custom processing algorithm to increase the image contrast then image clustering.
To validate the increment of the image contrast we utilize the Histogram chart. The original image of the investigated liver tissue at spectral wavelenght $(900 \mathrm{~nm})$, is presented in Fig. 7(a) that is drawn in the histogram chart with green bars. Then, the enhanced image displayed in Fig. 7(b) was clearly highlighted the stretch of the histogram bars drawn in purple, as illustrated in Fig. 7(c). Furthermore, we apply the same procedure on the additional experimental investigation liver tissue samples, where Sample \#4 and Sample \#5 after ablation and slicing had been displayed in Fig. A5 and Fig. A6, respectively in the Appendix.

Fig. 8 represents the custom algorithm processing, where the selected optimum spectral image $(900 \mathrm{~nm})$ as the original image. Which could discriminate between (normal / ablated) and additionally between (thermal / ablated) regions, as displayed in Fig. 8(a). Increasing the image contrast utilizing the "Top-hat Transform" and "Bottom-hat Transform", as shown in Fig. 8(b) and Fig. 8(c), respectively. The image outcome in the Fig. 8(d) is the high contrast image. Next, this high contrast image with the "Watershed segmentation", as shown in Fig. 8(e) was used. Finally, there are the delineation of the ablation region in red contour and the thermally affected region in blue contour, as displayed in Fig. 8(f). Moreover, to validate the custom algorithm processing, we apply the same steps on the additional experimental investigation liver tissue samples, where Sample \#4 and Sample \#5 after ablation and slicing had been displayed in Fig. A7 and Fig. A8, respectively in the Appendix.
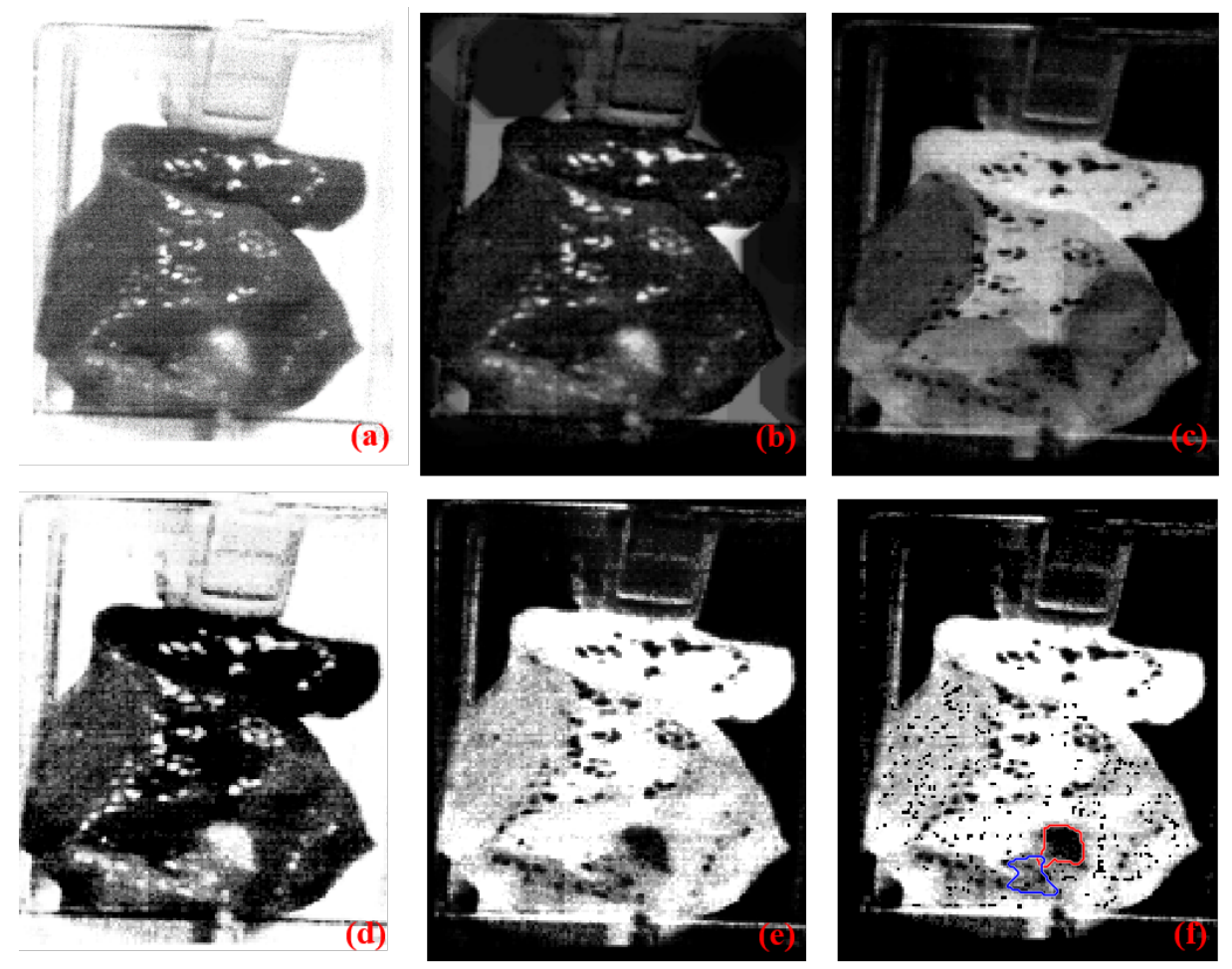

Fig. 8 The investigated liver tissue Sample \#1; (a) the acquired hyperspectral image at $900 \mathrm{~nm}$; (b) the image contrast enhancement after top-hat transforms; (c) the image contrast enhancement after bottom-hat transforms; (d) the enhanced contrast image; (e) the final complement enhancement image; (f) the delineation contour for the ablation and thermally affected region in red and blue, respectively. 


\section{Discussion}

The non-invasive thermal ablation such as (RFA, MWA, and Laser Ablation) is the leading therapeutic equipment for un-resection liver tumors. The thermal observation with the well-chosen image-guided system is vital to achieving a fruitful removal of tumors with the least possible thermal damage of liver tissues [75-78].

The presented approach setup utilizing HS camera aims to measure the diffuse reflectance $\left(R_{d}\right)$ of the selected regions (normal, thermal, and ablated) for the investigated ex vivo liver Sample \#1. The scanned HS image cube over the spectral range (400 1000 nm) shows a different spectral signature for each region, as displayed in Fig. 4.

To reduce the time of image processing for the investigated cube, we select from the 447 spectral channel, ten images in the wavelength range $(450 \sim 900 \mathrm{~nm})$ with $50 \mathrm{~nm}$ resolution. Although, from visual inspection, it was clear that the wavelength range $(550 \sim 650 \mathrm{~nm})$ was highly identifying between the normal and the thermal region. However, wavelength $(850 \sim 950 \mathrm{~nm}$ ) highlights more the contrast between the normal and ablated regions, as liver Sample \#1 illustrated in Fig. 5, liver Sample \#4 in Fig. A3, and liver Sample \#5 in Fig. A4 at the Appendix.

Demonstrating these results by utilizing the histogram in the appearance of a bar graph to highlight the contrast between the investigated ex vivo liver sample regions (normal, thermal, and ablated). Wavelength $(600 \mathrm{~nm})$ represents a high contrast between the normal and the thermal regions. Contrariwise, at a wavelength $(900 \mathrm{~nm})$ represents high contrast between the normal and the ablated regions, as shown in Fig. 6.

Next, to verify the experimental results we exploited the statistical analysis of the measured diffuse reflectance $\left(R_{d}\right)$ for the investigated samples. Which, demonstrates a high sample variance $\left(\hat{S}_{v}\right)$ between normal and thermal regions at a wavelength $(600 \mathrm{~nm})$. However, the wavelength $(900 \mathrm{~nm})$ shows a high variance between both the normal / ablated and ablated / thermal regions, as represented in Table 1.

Selecting the optimum spectral image $(900 \mathrm{~nm})$ which could discriminate between (normal/ablated) regions, additionally between (thermal / ablated) regions to the original image utilized with the custom processing algorithm to increase the image contrast then image clustering.

To verify the enhancement of the image contrast we utilize the histogram chart, as shown in Fig. 7. The original image of the investigated liver tissue at spectral image $(900 \mathrm{~nm})$, is presented in Fig. 7(a) that is was drawn in the histogram chart with green bars. The enhanced image displayed in Fig. 7(b) was clearly highlighted the stretch of the histogram bars drawn in purple, as illustrated in Fig. 7(c). Furthermore, for more validation to the algorithm steps, we apply the same procedure on the additional experimental investigation liver tissue samples, where Sample \#4 and Sample \#5 after ablation and slicing had been displayed in Fig. A5 and Fig. A6, respectively in the Appendix.

The optimum spectral image at $(900 \mathrm{~nm})$ was selected and processed by the custom algorithm, to discriminate mainly between (Normal / Ablated), then classify (Thermal / Ablated) regions. To enhance the image contrast, we exploited the "Top-hat Transform" and "Bottom-hat Transform", as shown in Fig. 8(b) and Fig. 8(c), respectively. The outcome improved image, as presented in Fig. 8(d) was processed utilizing the "Watershed segmentation", as shown in Fig. 8(e). Furthermore, the delineation of the ablation and thermally affected regions were displayed in Fig. 8(f) and identified with red and blue contours, respectively. Finally, for more validation to the algorithm process, we repeat the experimental investigation on additional liver samples, as shown in Fig. A7 and Fig. A8 in the Appendix, for sample \#4 and sample \#5, respectively.

\section{Conclusion}

In brief, the represented approach reveals the hyperspectral camera capabilities to provide precise information for surgeons by early delineation to the detected thermal effects to avoid overheating ablation. This overheating may lead to cell necrosis due to the inaccuracy of the exploratory modality used, or the lack of experience. Hyperspectral imaging is a prevailing tool in perception the thermal ablation with the least time, however, it cost too much in regards to the commercial CCD camera and it can't work progressively as the spectral cube consumes more time for image analysis over the 447 frames and image processing. To reduce the time expenses for HSI data processing we were able to identify a spectral band, centered at $600 \mathrm{~nm}$, to discriminate between the normal and the thermally ablated regions Moreover, at the spectral band centered at $900 \mathrm{~nm}$ a high contrast was achieved between the normal and the ablated regions. Therefore, it would be possible in the future work to provide direct feedback on the resection edges to the specialist during surgery. Along these lines, the subsequent stage is to approve the procedure as an edge evaluation method during liver tumor surgery.

\section{Disclosures}

The authors declare and state that the work was affirmed by the Ethics Committee of Ain Shams university Faculty of Medicine, Cairo, Egypt. We confirm that this work is original and has not been published elsewhere nor is it currently under consideration for publication elsewhere. The authors declare and state that they have all the research data and all the materials are available. The authors declare and state that they have no competing interests. The authors declare that there are no funders neither no one had any role in the design of the study; in the collection, analyses, or interpretation of data; in the writing of the manuscript, or in the decision 
to publish the results. The authors declare and stated that

they have not Acknowledgements.

\section{References}

1. S. Morini, G. Carpino, S. Carotti, and E. Gaudio, "Anatomy and Embryology of the Liver," Chapter in Liver Diseases, F. Radu-Ionita, N. T. Pyrsopoulos, M. Jinga, I. C. Tintoiu, Z. Sun, and E. Bontas (Eds.), Springer International Publishing, Cham, 3-16 (2020).

2. M. W. Robinson, C. Harmon, and C. O. Farrelly, "Liver immunology and its role in in fl ammation and homeostasis," Cellular \& Molecular Immunology 13, 267-276 (2016).

3. C. Review, "Global, Regional, and National Cancer Incidence, Mortality, Years of Life Lost, Years Lived With Disability, and Disability- Adjusted Life-years for 32 Cancer Groups, 1990 to 2015 A Systematic Analysis for the Global Burden of Disease Study," JAMA Oncology 3(4), 524-548 (2017).

4. J. Ferlay, H. R. Shin, F. Bray, D. Forman, C. Mathers, and D. M. Parkin, "Estimates of worldwide burden of cancer in 2008: GLOBOCAN 2008," International Journal of Cancer 127(12), 2893-2917 (2010).

5. Y. F. Liaw, “Antiviral therapy of chronic hepatitis B: Opportunities and challenges in Asia,” Journal of Hepatology 51(2), 403-410 (2009).

6. P. Ferenci, M. Fried, D. Labrecque, J. Bruix, M. Sherman, M. Omata, J. Heathcote, T. Piratsivuth, M. Kew, J. A. Otegbayo, S. S. Zheng, S. Sarin, S. S. Hamid, S. B. Modawi, W. Fleig, S. Fedail, A. Thomson, A. Khan, P. Malfertheiner, G. Lau, F. J. Carillo, J. Krabshuis, and A. Le Mair, "Hepatocellular carcinoma (HCC): A global perspective," Journal of Clinical Gastroenterology 44(4) 239-245 (2010).

7. S. Abd-Elsalam, N. Elwan, H. Soliman, D. Ziada, W. Elkhalawany, M. Salama, N. Hawash, M. Arafa, R. Badawi, and W. M. Shehata, "Epidemiology of liver cancer in Nile delta over a decade: A single-center study," South Asian Journal of Cancer 7(1), 24 (2018).

8. T. Hennedige, S. K. Venkatesh, "Imaging of hepatocellular carcinoma: diagnosis, staging and treatment monitoring," Cancer Imaging 12(3), 530 (2012).

9. T. Longerich, K. Breuhahn, and P. Schirmacher, "Molecular pathology of liver tumors," in Molecular Surgical Pathology, L. Cheng, J. Eble (Eds), Springer, New York, NY, 43-63 (2013). ISBN: 978-1-4614-4899-0.

10. J. Ferlay, I. Soerjomataram, R. Dikshit, S. Eser, C. Mathers, M. Rebelo, D.M. Parkin, D. Forman, and F. Bray, "Cancer incidence and mortality worldwide: sources, methods and major patterns in GLOBOCAN 2012," International Journal of Cancer 136(5), E359-E386 (2015).

11. W. M. Rashed, M. A. M. Kandeil, M. O. Mahmoud, and S. Ezzat, "Hepatocellular Carcinoma (HCC) in Egypt: A comprehensive overview," Journal of the Egyptian National Cancer Institute 32(1), 1-11 (2020).

12. M. J. Englesbe, S. P. Patel, K. He, R. J. Lynch, D. E. Schaubel, C. Harbaugh, S. A. Holcombe, S. C. Wang, D. L. Segev, and C. J. Sonnenday, "Sarcopenia and mortality after liver transplantation," Journal of the American College of Surgeons 211(2), 271-278 (2010).

13. H. Liao, "Integrated diagnostic and therapeutic techniques: Toward an intelligent medical system," Computerized Medical Imaging and Graphics:The Official Journal of the Computerized Medical Imaging Society 38(5), 421 (2014).

14. N. Sanai, M.-Y. Polley, M. W. McDermott, A. T. Parsa, and M. S. Berger, "An extent of resection threshold for newly diagnosed glioblastomas," Journal of Neurosurgery 115, 3-8 (2011).

15. B. K. P. Goh, J.-Y. Teo, C.-Y. Chan, S.-Y. Lee, P.-C. Cheow, P. K. H. Chow, L. L. P. J. Ooi, and A. Y. F. Chung, "Evolution of laparoscopic liver resection at Singapore General Hospital: a nine-year experience of 195 consecutive resections," Singapore Medical Journal 58(12), 708 (2017).

16. A. Forner, M. Gilabert, J. Bruix, and J. L. Raoul, “Treatment of intermediate-stage hepatocellular carcinoma," Nature Reviews Clinical Oncology 11, 525-535 (2014).

17. E. Tanis, J. W. Spliethoff, D. J. Evers, and G. C. Langhout, "Real-time in vivo assessment of radiofrequency ablation of human colorectal liver metastases using diffuse reflectance spectroscopy," European Journal of Surgical Oncology 42(2), 251-259 (2020).

18. T. Livraghi, H. Mäkisalo, and P. Line, “Treatment options in hepatocellular carcinoma today," Scandinavian Journal of Surgery 100(1), 22-29 (2011).

19. S. G. Nour, "MRI-Guided Laser Ablation of Liver Tumors," in Primary and Metastatic Liver Tumors, K. Cardona, S. Maithel (Eds), Springer, Cham, 323-332 (2018).

20. G. Giuseppe, D. Costanzo, C. Hospital, and M. Guarracino, Image-guided Laser Ablation, Springer, Cham (2020). ISBN: 978-3-030-21747-1.

21. C. L. Brace, Microwave tissue ablation: Biophysics, technology, and applications, Critical Reviews in Biomedical Engineering 38(1), 65-78 (2010).

22. B. Imene, B. Farid, and B. Khaoula, "CT-guided microwave liver tumors ablation and automatic adjustment of frequency," Averroes European Medical Journal 2(1), (2018). 
23. J. Macdonell, N. Patel, S. Rubino, G. Ghoshal, G. Fischer, E.C. Burdette, R. Hwang, and J. G. Pilitsis, "Magnetic resonance-guided interstitial high-intensity focused ultrasound for brain tumor ablation," 44(2), 1-6 (2018).

24. H. Zhi-Yu, L. Ping, Y. Xiao-Ling, C. Zhi-Gang, L. Fang-Yi, and Y. Jie, "A clinical study of thermal monitoring techniques of ultrasound-guided microwave ablation for hepatocellular carcinoma in high-risk locations," Scientific Reports 7, 41246 (2017).

25. H. Takahashi, M. Akyuz, E. Aksoy, K. Karabulut, and Eren Berber, "Local recurrence after laparoscopic radiofrequency ablation of malignant liver tumors : Results of a contemporary series," Journal of Surgical Oncology 115(7), 830-834 (2017).

26. T. R. Fosnight, F. M. Hooi, R. D. Keil, A. P. Ross, S. Subramanian, T. G. Akinyi, J. K. Killin, P. G. Barthe, S. M. Rudich, S. A. Ahmad, M. B. Rao, and T. G. Mast, "Echo decorrelation imaging of rabbit liver and VX2 tumor during in vivo ultrasound ablation," Ultrasound in Medicine \& Biology 43(1), 176-186 (2017).

27. T. J. Vogl, A. Dommermuth, B. Heinle, N.-E. A. Nour-Eldin, T. Lehnert, K. Eichler, S. Zangos, W. O. Bechstein, and N. N. N. Naguib, "Colorectal Cancer Liver Metastases: Long-Term Survival and Progression-Free Survival After Thermal Ablation Using Magnetic Resonance-Guided Laser-Induced Interstitial Thermotherapy in 594 Patients Analysis of Prognostic Factors," Investigative Radiology 49(1), 48-56 (2014).

28. J. Paul, T. J. Vogl, and A. Chacko, "Dual energy computed tomography thermometry during hepatic microwave ablation in an ex-vivo porcine model," Physica Medica 31(7), 683-691 (2015).

29. M. De Landro, J. Ianniello, M. Yon, A. Wolf, B. Quesson, E. Schena, and P. Saccomandi, "Fiber bragg grating sensors for performance evaluation of fast magnetic resonance thermometry on synthetic phantom," Sensors 20(22), $1-17$ (2020).

30. L. Feng, S. Zhu, F. Liu, Y. He, Y. Bao, and C. Zhang, "Hyperspectral imaging for seed quality and safety inspection: A review," Plant Methods 15, 91 (2019).

31. P. Martín-Mateos, F. U. Khan, and O. E. Bonilla-Manrique, "Direct hyperspectral dual-comb imaging," Optica 7(3), 199-202 (2020).

32. R. Abdlaty, Q. Fang, "Acousto-optic tunable filter-based hyperspectral imaging system characterization," SPIE Proceeding 10870, 108700Y (2019).

33. M. A. Calin, A. C. Calin, and D. N. Nicolae, "Application of airborne and spaceborne hyperspectral imaging techniques for atmospheric research: past, present, and future,” Applied Spectroscopy Reviews 56(4), 1-35 (2020).

34. G. Polder, A. Gowen, The hype in spectral imaging, Journal of Spectral Imaging 9, (2020).

35. D. Inamdar, M. Kalacska, G. Leblanc, and J. P. Arroyo-Mora, "Characterizing and mitigating sensor generated spatial correlations in airborne hyperspectral imaging data," Remote Sensing 12(4), 641 (2020).

36. X. Jin, H. Memon, W. Tian, Q. Yin, X. Zhan, and C. Zhu, "Spectral characterization and discrimination of synthetic fibers with near-infrared hyperspectral imaging system," Applied Optics 56(12), 3570-3576 (2017).

37. E. Bonah, X. Huang, J. H. Aheto, and R. Osae, "Application of hyperspectral imaging as a nondestructive technique for foodborne pathogen detection and characterization," Foodborne Pathogens and Disease 16(10), 712-722 (2019).

38. M. L. Pieper, D. Manolakis, E. Truslow, T. W. Cooley, M. Brueggeman, J. Jacobson, and A. Weisner, "Performance limitations of temperature-emissivity separation techniques in long-wave infrared hyperspectral imaging applications," Optical Engineering 56(8), 81804 (2017).

39. J. Deal, S. Mayes, C. Browning, S. Hill, P. Rider, C. Boudreaux, T. C. Rich, and S. J. Leavesley, "Identifying molecular contributors to autofluorescence of neoplastic and normal colon sections using excitation-scanning hyperspectral imaging," Journal of Biomedical Optics 24(2), 021207 (2018).

40. M. De Landro, I.E. García-Molina, M. Barberio, E. Felli, V. Agnus, M. Pizzicannella, M. Diana, E. Zappa, and P. Saccomandi, "Hyperspectral Imagery for Assessing Laser-Induced Thermal State Change in Liver," Sensors 21(2), 643 (2021).

41. M. De Landro, M. Barberio, E. Felli, V. Agnus, M. Pizzicannella, M. DIana, and P. Saccomandi, "Hyperspectral imaging system for monitoring laser-induced thermal damage in gastric mucosa," In 2020 IEEE International Symposium on Medical Measurements and Applications (MeMeA), 1-6 (2020).

42. R. Abdlaty, J. Haywar, T. Farrell, and Q. Fang, "Skin Erythema and Pigmentation Optical Assessment Techniques," Photodiagnosis and Photodynamic Therapy 33, 102127 (2020).

43. M. H. Aref, I. H. Aboughaleb, and Y. H. El-Sharkawy, "Custom optical imaging system for ex-vivo breast cancer detection based on spectral signature," Surgical Oncology 35, 547-555 (2020).

44. M. H. Aref, A. M. Youssef, I. H. Aboughaleb, and Y. H. El-sharkawy, "Characterization of Normal and Malignant Breast Tissues utilizing Hyperspectral Images and Associated Differential Spectrum Algorithm," Journal of Biomedical Photonics \& Engineering 7(2), 020302 (2021).

45. I. H. Aboughaleb, M. H. Aref, and Y. H. El-Sharkawy, "Hyperspectral imaging for diagnosis and detection of exvivo breast cancer," Photodiagnosis and Photodynamic Therapy 31, 101922 (2020).

46. M. Aref, I. H. Aboughaleb, A.-B. Youssef, and Y. El-Sharkawy, "Novel Approach Exploiting the Hyperspectral Imaging System for Breast Cancer Therapy and Diagnosis," Archives of Breast Cancer 7(4), 189-201 (2020). 
47. S. Ortega, M. Halicek, H. Fabelo, R. Camacho, M. d. 1. L. Plaza, F. Godtliebsen, G. M. Callicó, and B. Fei, "Hyperspectral Imaging for the Detection of Glioblastoma Tumor Cells in H\&E Slides Using Convolutional Neural Networks," Sensors 20, 1911 (2020).

48. H. Fabelo, S. Ortega, R. Lazcano, D. Madroñal, G. M. Callicó, E. Juárez, R. Salvador, D. Bulters, H. Bulstrode, A. Szolna, J. F. Piñeiro, C. Sosa, A. J. O’Shanahan, S. Bisshopp, M. Hernández, J. Morera, D. Ravi, B. R. Kiran, A. Vega, A. Báez-Quevedo, G.-Z. Yang, B. Stanciulescu, and R. Sarmiento, “An Intraoperative Visualization System Using Hyperspectral Imaging to Aid in Brain," Sensors 18(2), 430 (2018).

49. M. H. Fouad Aref, A. A. R. Sharawi, and Y. H. El-Sharkawy, "Delineation of the Arm Blood Vessels Utilizing Hyperspectral Imaging to Assist with Phlebotomy for Exploiting the Cutaneous Tissue Oxygen Concentration," Photodiagnosis and Photodynamic Therapy 33, 102190 (2021).

50. P.-C. Chen, W.-C. Lin, "Spectral-profile-based algorithm for hemoglobin oxygen saturation determination from diffuse reflectance spectra," Biomedical Optics Express 2(5), 1082-1096 (2011).

51. M. W. Wukitsch, "Pulse oximetry: Historical review and Ohmeda functional analysis," International Journal of Clinical Monitoring and Computing 4, 161-166 (1987).

52. V. Tuchin, "Tissue Optics and Photonics: Light-Tissue Interaction II," Journal of Biomedical Photonics \& Engineering 2(3), 030201 (2016).

53. V. V Tuchin, "Tissue Optics and Photonics: Biological Tissue Structures," Journal of Biomedical Photonics \& Engineering 1(1), 3-21 (2015).

54. M. H. Aref, "Biomedical Research and Clinical Reviews," Biomedical Research and Clinical Reviews 1, 1-13 (2020).

55. M. H. Aref, I. H. Aboughaleb, and Y. H. El-sharkawy, "Photodiagnosis and Photodynamic Therapy Tissue characterization utilizing hyperspectral imaging for liver thermal ablation," Photodiagnosis and Photodynamic Therapy 31, 101899 (2020).

56. M. H. Aref, I. H. Aboughaleb, A.-B. M. Youssef, and Y. H. El-Sharkawy, "Hyperspectral image-based analysis of thermal damage for ex-vivo bovine liver utilizing radiofrequency ablation," Surgical Oncology 38, 101564 (2021).

57. A. Robles-Kelly, C. P. Huynh (Eds.), Imaging Spectroscopy for Scene Analysis, Springer, London (2013). ISBN: 978-1-4471-4652-0.

58. J. M. Bioucas-Dias, A. Plaza, G. Camps-Valls, P. Scheunders, N. Nasrabadi, and J. Chanussot, "Hyperspectral remote sensing data analysis and future challenges," IEEE Geoscience and remote sensing magazine 1(2), 6-36 (2013).

59. T. Adão, J. Hruška, L. Pádua, J. Bessa, E. Peres, R. Morais, and J. J. Sousa, "Hyperspectral imaging: A review on UAV-based sensors, data processing and applications for agriculture and forestry," Remote Sensing 9(11), 1110 (2017).

60. S. S. M. Noor, K. Michael, S. Marshall, and J. Ren, "Hyperspectral image enhancement and mixture deep-learning classification of corneal epithelium injuries," Sensors 17(11), 2644 (2017).

61. N. Dhanachandra, K. Manglem, and Y. J. Chanu, "Image Segmentation Using K-means Clustering Algorithm and Subtractive Clustering Algorithm," Procedia Computer Science 54, 764-771 (2015).

62. A. Janßen, P. Wan, “k-means clustering of extremes,” Electronic Journal of Statistics 14(1), 1211-1233 (2020)

63. A. Badawi, M. Bilal, "High-level synthesis of online k-means clustering hardware for a real-time image processing pipeline," Journal of Imaging 5(3), 38 (2019).

64. A. Gulhane, P. L. Paikrao, and D. S. Chaudhari, "A Review of Image Data Clustering Techniques," International Journal of Soft Computing and Engineering 2(1), 212-215 (2019).

65. M. A. Hamdi, "Modified Algorithm marker-controlled watershed transform for Image segmentation Based on Curvelet Threshold," Canadian Journal on Image Processing and Computer Vision 2(8), 88-91 (2008).

66. S. Derivaux, G. Forestier, C. Wemmert, and S. Lefèvre, "Supervised image segmentation using watershed transform, fuzzy classification and evolutionary computation," Pattern Recognition Letters 31(15), 2364-2374 (2010).

67. Y. Zhao, J. Liu, H. Li, and G. Li, "Improved Watershed Algorithm for Dowels Image Segmentation," In 2008 7th World Congress on Intelligent Control and Automation, 7644-7648 (2008).

68. X. Fengying, J. Zhiguo, and Z. Fugen, "Immune cell image segmentation based on mathematical morphology," Journal of Image and Graphics 7(11), 1119-1122 (2002).

69. H. P. Ng, S. H. Ong, K. W. C. Foong, P. S. Goh, and W. L. Nowinski, "Medical Image Segmentation Using K-Means Clustering and Improved Watershed Algorithm," 2006 IEEE Southwest Symposium on Image Analysis and Interpretation, 61-65 (2006).

70. S. Liu, L. Zeng, B. Liu, and Y. Fang, "Separating Algorithm for Overlapping Granule Images," Computer Engineering 28(2), 198-199 (2002).

71. X. Yang, S. Zhan, D. Xie, H. Zhao, and T. Kurihara, "Hierarchical prostate MRI segmentation via level set clustering with shape prior," Neurocomputing 257, 154-163 (2017).

72. J. C. Dunn, "A fuzzy relative of the ISODATA process and its use in detecting compact well-separated clusters," Journal of Cybernetics 3(3), 32-57 (1973).

73. C. Kalyani, K. Ramudu, and G. R. Reddy, “A review on optimized k-means and FCM clustering techniques for biomedical image segmentation using level set formulation,” Biomedical Research 29(20), 3660-3668 (2018). 
74. V. Kumar, P. Gupta, "Importance of Statistical Measures in Digital Image Processing," International Journal of Emerging Technology and Advanced Engineering 2(8), 56-62 (2012).

75. H. Wu, L. R. Wilkins, N. P. Ziats, J. R. Haaga, and A. A. Exner, "Real-time monitoring of radiofrequency ablation and postablation assessment: Accuracy of contrast-enhanced US in experimental Rat liver model," Radiology 270(1), 107-116 (2014).

76. P. Wiggermann, K. Brünn, J. Rennert, M. Loss, H. Wobser, A. G. Schreyer, C. Stroszczynski, and E. M. Jung, "Monitoring during hepatic radiofrequency ablation (RFA): Comparison of real-time ultrasound elastography (RTE) and contrast-enhanced ultrasound (CEUS): First clinical results of 25 patients," Ultraschall in der Medizin-European Journal of Ultrasound 34(06), 590-594 (2013).

77. Y. Z. Zhang, T. Xu, H. Y. Gong, C. Y. Li, X. H. Ye, H. J. Lin, M. P. Shen, Y. Duan, T. Yang, and X. H. Wu, "Application of high-resolution ultrasound, real-time elastography, and contrast-enhanced ultrasound in differentiating solid thyroid nodules," Medicine 95(45), e5329 (2016).

78. S. Swierczynski, F. Primavesi, E. Klieser, T. Kiesslich, T. Jäger, R. Illig, D. Neureiter, D. Ofner, and S. Stättner, "382. Thermographic monitoring of radiofrequency and microwave ablation in a perfused porcine liver model," European Journal of Surgical Oncology 40(11), S147 (2014). 